\title{
Image-Free Handheld Robot for Total Knee Arthroplasty Improves Early Functional Recovery Compared with Conventional Total Knee Arthroplasty
}

Kazu Matsumoto ( $\square$ mkazuu@gifu-u.ac.jp )

Gifu University

Hiroyasu Ogawa

Ogaki Tokushukai Hospital

Hiroki Yoshioka

Gifu University

Yutaka Nakamura

Ogaki Tokushukai Hospital

Haruhiko Akiyama

Gifu University

\section{Research Article}

Keywords: robot, conventional, total knee arthroplasty, range of motion, muscle strength, Navio

Posted Date: November 16th, 2021

DOI: https://doi.org/10.21203/rs.3.rs-1048544/v1

License: (a) (1) This work is licensed under a Creative Commons Attribution 4.0 International License.

Read Full License 


\section{Abstract}

This study aimed to examine whether it is advantageous in robotic-assisted TKA (RA-TKA) compared with conventional TKA throughout a 14 postoperative days (PODs). A total of 113 knees (100 patients) were reviewed and divided into the control group (55 knees) and the RA-TKA group (58 knees). We assessed postoperative pain intensity using a visual analogue scale at rest (rVAS) and during movement (mVAS), evaluated lower extremity functional recovery through quadriceps muscle strength and knee range of motion (ROM), preoperatively and on $\mathrm{PODs} 3,7,10$, and 14 . We also assessed the rescue analgesia intake and postoperative implant coronal alignment. The mean rVAS and mVAS scores did not differ significantly between the two groups. Muscle strength recovery was significantly faster in the RATKA group than in the control group on every PODs. ROM recovery was better in the RA-TKA group than in the control group on POD 10. The amount of postoperative analgesia was significantly lower in the RATKA group than in the control group. Attainment of a $\beta$ angle $<2^{\circ}$ significantly better in RA-TKA. This study demonstrated better functional recovery in RA-TKA, particularly for muscle strength and ROM. RATKA reduced rescue drug intake and provided better implant positioning.

\section{Introduction}

Patient satisfaction is an important outcome which will still be improved for total knee arthroplasty (TKA) since its levels are between $80 \%$ and $90 \%$ [1]. The explanations for patient dissatisfaction include lower implant survivorship, poorer function, and need for revision surgery, resulting from component malalignmentor soft tissue imbalance [2-7]. Recently, robotic-assisted TKA (RA-TKA) was introduced within the orthopaedic field, and this technology brings a possible solution to the issues faced byconventional TKA.

The robotic systems for TKA are mainly subcategorised intothree main subtypes: passive, semi-active, andactive robotic systems [8]. Semi-active robotic systems enable theupkeep of the general control of bone resection and implantpositioning. There are two sorts of semi-active robotic systems: imagedependent and imageless. TheMako Robotic Arm Interactive Orthopaedic system (StrykerLtd, Kalamazoo, $\mathrm{MI}$, USA) is an image-guidedsemi-active robotic system for RA-TKA. On the other hand, theNavioSurgical System (Smith \& Nephew, Inc., Memphis, TN, USA) is an image-freehandheld robotic sculpting system introduced in knee arthroplasties in 2017 [9].

Robotic systems are attaina more accurate implant alignment compared with conventional methods. Several reports have shown that robotic-assisted surgical procedures improve prosthetic alignment and position. Hampp et al. [10] conducted a cadaveric study to look at the accuracy of component positioning in Mako RA-TKA compared with manual TKA, and RA-TKA achieved a more accurate implant positioning. Bollars et al. [11] examined the limb alignment and orientation of components in TKA using the Navioimage-free handheld robotic sculpting system. They concluded that the Navio group showed significantly fewer deviations compared with the conventional technique.Therefore, RA-TKA is advantageous for achieving accurate implant alignment. 
Improved accuracy of implant positioning in RA-TKA may enhancepostoperative functionaloutcomes compared to conventional jig-based methods. Kayani et al. [12] conducted a prospective study to check early functional recovery and hospital discharge inpatients undergoing conventional jig-based TKA compared with Mako RA-TKA. They found that RA-TKA reduced pain andimproved early functional recovery within the primary3 days compared to conventional TKA. Several studies have suggested greater functionalimprovements using the Mako system as measured by the Knee Society Score andtherefore the Western Ontario andMcMaster Universities Osteoarthritis Indexin the short term [13, 14]. However, there arelimited data about the early postoperative period, like 14 days after surgery, on the employment of NavioRA-TKA.

Therefore, this study focused on the postoperative period and examined functional recovery, postoperative pain, implant alignment, and rescue dose in NavioRA-TKA compared with conventional TKA. We hypothesise that Navio RA-TKA provides better functional recovery than conventional TKA.

\section{Results}

\section{Demographic and clinical data}

The preoperative backgrounds of patients are shown in Table 1. There were no significant differences in age, height, body weight, BMI, and FTA between the RA-KTA and control groups.

\section{Postoperative pain}

Postoperative pain intensity was measuredusing 100-mm rVASand mVASscales. The mean rVAS (Figure 1A) and mean mVAS (Figure 1B) scores did not differ significantly between the two groups throughout the 14-day postoperative period.

\section{Postoperative functional recovery}

Lower extremity functional recovery was evaluated by examining the MS using a muscle tester. MS recovery wassignificantly faster in the RA-TKA group than in the control group on PODs3 $(p=0.0266)$, $7(p=0.0104), 10(p=0.0384)$, and $14(p=0.0068)$ (Figure 2$)$. We also examined the postoperative knee ROM (Figure 3). The postoperative ROM recovery, which was indicated by \% ROM, was better in the RA-TKA group than in the control group. There were no statistically significant differences between the two groups on PODs3 $(p=0.0789), 7(p=0.0899)$, and $14(p=0.0852)$. However, there was a significant improvement for ROM recovery in the RA-TKA group on POD10 $(p=0.0231)$.

We further examined the femoral circumference to evaluate the soft tissue swelling. The femoral circumference (\%) was comparable in both groups throughout the postoperative period (Figure 4).

\section{Rescue dose}


The number of times a rescue drug was administered for postoperative painwas significantly smaller in the RA-TKA group than in the control group (RA-TKA:4.12 \pm 7.26 , control:7.98 $\pm 12.1, p=0.0399$ ) until POD14.

\section{Radiographic evaluation}

The mean postoperative coronal alignment of the femoral component was comparable between the two groups ( $p=0.4232$ ), with an aangle of $92.2^{\circ} \pm 2.1^{\circ}$ in the control group and $92.3^{\circ} \pm 1.1^{\circ}$ in the RA-TKA group (Table 2). Proper coronal alignment of the femoral component, defined by an a deviation angle $<2^{\circ}$ relative to the mechanical axis of the knee, was achieved in $92.6 \%$ of knees in the control group and $92.9 \%$ in the RA-TKA group (Figure 5A and Table 2), and there were no significant differences between the groups $(p=0.9579)$ (Table 2$)$.

The mean postoperative coronal alignment of the tibial component was comparable between the two groups $(p=0.4427)$, with a $\beta$ angle of $89.6^{\circ} \pm 2.2^{\circ}$ for the control group and $89.6^{\circ} \pm 1.0^{\circ}$ for the RA-TKA group (Table 2). Proper coronal alignment of the tibial component, defined by a $\beta$ deviation angle $<2^{\circ}$ relative to the mechanical axis of the knee, was achieved in $72.2 \%$ of knees in the control group and $96.4 \%$ in the RA-TKA group (Figure 5 and Table 2). There was a significant difference between the two groups $(p=0.0004)$ (Table 2$)$.

\section{Discussion}

The most important finding of the current study was that an image-free handheld RA-TKA allowed better functional recovery, including muscle strength and ROM recovery, and required less analgesiacompared with jig-based conventional TKA. Furthermore, RA-TKA decreased the deviations of the implant and achieved significantly accurate alignment, especially within the tibial component.

Robotic systems have been introduced to realisea more accurate implant alignment compared with conventional methods.Fully active robotic systems were the primary robot systems employed within the orthopaedic field since 1992 [19]. This technique has demonstrated improvements in radiologicaloutcomes but also presented the disadvantages of the short-term complications like superficial infection,patellar dislocation,supracondylar or patellar fracture, and commonperoneal injury [20]. Recently, semi-active robot systems are increasingly utilized in TKA. Thistechnique allows the surgeon to guidethe robotic arm to perform the bonypreparation, and thereforerequires constant input forthe procedure to be completed.Semi-active robotic systems enable surgeons tohavethe overall control for bone resection and implantpositioning. The image-freehandheld robotic sculpting system, Navio, was introduced in knee arthroplasties in 2017 [9]. This system does not require preoperative CT imaging, which is an advantageous point. Intraoperatively,a probe is used tomap out the bony anatomy.

Little information is available on early postoperative functional recovery in semi-active robotic systems. Kayani et al. [12] compared early postoperative functional outcomes between conventional jigbasedTKAand Mako RA-TKA and found that the RA-TKA group showed reduced pain and decreased 
analgesic requirements at POD 3. Bhimani et al. [21] retrospectively reviewed 140patients undergoing Mako RA-TKAand found that RA-TKA resulted in lower pain levels at both rest and movement at 2 and 6 weeks postoperatively andrequiredless opioid medication. In this study, we reviewed postoperative pain for 14 days in NavioRA-TKA but did not find significant differences in postoperative pain at rest or during movement. However, the number of rescue drugs needed was significantly lower in the RA-TKA group compared with the control group, suggesting that RA-TKA may reduce postoperative pain.

Furthermore, the recovery of MS was significantly better in the RA-TKA group than in the control group. There have been no previous reports evaluating quadriceps MS directly in RA-TKA. Kayani et al. [12] examined 40 patients undergoing conventionaljig-based TKA and 40 patients who underwent Mako RATKA. Straight leg raise was achieved faster in RA-TKA than in conventional TKA. Additionally, recovery of ROM tended to be better in the RA-TKA group than in the control group during the 14-day postoperative period in this study. Kayani et al. [12] reported improved maximum knee flexion in Mako RA-TKA compared with the conventional TKA group at discharge. Meanwhile, Naziri et al. [22] reported that theMako RA-TKA cohort demonstrated improved ROM compared to the traditional TKAcohort at 90 days postoperatively. On the other hand, Jeon et al. [23] reported no significant differencesinROM between the ROBODOC RA-TKA and conventional TKA groups at 129 months postoperatively. Similarly, Cho et al. [24] also reported no significant differences in ROM between the ROBODOC RA-TKA and conventional TKA groups after a mean follow-up of 11.0 years. Therefore, RA-TKA could be more advantageous for early functional recovery, especially for MS and ROM.

Several reports have reported that RA-TKA is related to fewer soft tissue injuries than conventional TKA $[25,26]$. Hamppet al.[25] conducted a cadaveric study and found thatRA-TKA was related toless soft tissue damage, including posterior cruciateligament injury,compared with conventional TKA.RA-TKA utilises haptic boundaries that limit theaction of the drill burr or sawblade for femoral and tibial resections, and real-time intraoperative data is obtained. This could minimise the extra soft tissue release and limit iatrogenic periarticular soft tissue injury compared with conventional procedures. In this study, we examined the femoral circumference to evaluate soft tissue swelling, and no significant differences were observed between the two groups. Overall, there is more evidence supporting that RA-TKA leads to less soft tissue damage in patients.

Many studies have reported the accuracy of implant positioning in semi-active robotic TKA. Kayani et al. [27] reported that Mako RA-TKA obtained better implant alignment than conventional TKA. Bollars et al. [11] examined the limb alignment and orientation of components in TKA employinga Navioimage-free handheld robotic sculpting system. They concluded that the Navio group showed significantly fewer deviations compared with the standard technique. Additionally, Kaneko et al. [28] reported that the rotational alignment of the tibial prosthesis was superior in Navio RA-TKA compared with conventional TKA using 3D-CT evaluation. During this study, the RA-TKA group showed a better accuracy of implant positioning, especially in tibial prostheses, compared with conventional TKA. Therefore, semi-active robotic TKA could be a reliable procedure for accurate implant positioning in TKA. 
This study had several limitations. First, this was a retrospective study, and a prospective randomised controlled study is needed. Second, the follow-up period was limited to 14 PODs, and long-term dataon functional outcomes or revisionrates were not available. Third, we evaluated implant accuracy using plain radiographs. CT scans could be more precise. Fourth, we did not consider the learning curve for the RA-TKA in this study. Kayani et al. [27] reported that seven cases of Mako RA-TKA were required for successful integration into the surgical workflow.

\section{Conclusions}

This study found better functional recovery in RA-TKA, including muscle strength and ROM recovery. RATKA reduced the need for rescue drug intake and provided better implant positioning. Therefore, our data support the utility and implementation of RA-TKA for orthopaedic surgeons.

\section{Methods}

\section{Participants}

From January 2018 to April 2021, we performed 123 primary TKAs using bi-cruciatestabilised (BCS) TKA (Journey II, Smith \& Nephew, Tokyo, Japan) in 110 patients at our institute. Patients with OA and knee varus deformities were included. The exclusion criteria were rheumatoid arthritis, osteonecrosis of the knee, posttraumatic $O A$, and valgus deformity of the knee. Ultimately, 113 knees (100 patients) were eligible for this study. We performed 55 TKAs (51 patients) using conventional extramedullary systems from January 2018 to December 2019, and 58 TKAs (49 patients) were performed using Navio RA-TKA from January 2020 to April 2021. Therefore, we divided the patients into two groups: the control group and the RA-TKA group. All study participants provided informed consent, andGifu University School of MedicinelnstitutionalEthics approved all procedures performed during this study (approval number: 2019238). The following methods were carried out in accordance with relevant guidelines and regulations.

\section{Surgical Procedures}

\section{Control group}

Extramedullary (EM)-guided TKA was performed in accordance with previously reported procedures [15, 16]. Before the operation, the patients were restrained against the operating table using surgical tape. The centre of the femoral head was marked with a rounded magnetic marker. The knee joint was exposed employing a medial parapatellar approach, and also the anterior and posterior cruciate ligaments were resected. The proximal tibia was cut using an EM guide. After resecting the tibial plateau, the surgeon followed the manufacturer's instructions regarding the use of the femoral EM guide. After setting the cutting guide sleeve, the EM rod was aligned with the magnetic marker from the Whiteside's line. After the distal femur was cut, gap balancing was performed to determine the rotation of the femoral component following soft-tissue balancing. The goal of the femoral alignment was $90^{\circ}$ to the mechanical axis within the frontal plane and $3^{\circ}$ flexion within the sagittal plane to avoid femoral cortex notching. After these 
procedures, additional osteophytes were removed, especially in the posterior aspect of the medial femoral condyle. The rotation of the tibial component made up our minds using the range-of-movement technique [17]. We then implanted the tibial and femoral components with cement.

\section{Robotic-assisted TKA group}

All procedures were performed within the same way as the control group before knee jointexposure. Then, two partially threaded pins were placed into the proximal tibia and distal femur for the tracking arrays of the robot system. The osteophytes were excised, and also the anterior and posterior cruciateligaments were resected before gathering data using the robot. The anatomical landmarks, including the hip centre, were registered in keeping with the manufacturer's procedure. The femur and tibiamorphologies were determined through mapping of the condylaranatomy bytracingthe surfaces with the probe tomake a virtual 3D model of the knee. Following this, varus and valgus stresses were applied duringthe full range of motion (ROM)to balance the dynamic soft tissue. The robotic systemcreated a graphical gap space through a full ROM. The surgeon can adjust the desired mechanical axis.In this study, we planned the femoral and tibial components to be perpendicular to the mechanical axis. Adjustments ofthe implant size, position, and insert thickness in all planes were adjusted tooptimise soft tissue balance and component tracking beforebone preparation. After planning the component, a high-speed 5-mm burr was wont to cut the distalfemur bone. This drill burr continuously moved the handpiece and switched on and offbecause the motorised burr moved in and out of the mapped cuttingarea. The pin holes for the proximal tibia saw guides were preparedwith a 5 -mm burr for the proximal tibia and a 2-mmburr for the distal femur. The saw guides were then fixedin an optimal position tomake bone cuts of the proximal tibia and anterior andposterior femur.After placing the trial components, balancing and componenttracking were checked with varus and valgusstress during full ROManda graphical representationof gap spacing.We then implanted the tibial and femoral components with cement.

\section{Outcome measurements}

The postoperative pain intensity was recorded by physical therapists employing a $100-\mathrm{mm}$ visual analogue scale (VAS; where $0=$ no pain and $100=$ worst imaginable pain) at rest (rVAS) and during movement (mVAS) preoperatively and on postoperative days (PODs) $3,7,10$, and 14 . They also evaluated lower extremity functional recovery by quadriceps muscle strength and therefore the knee's ROM.Adynamometer (Isoforce, OG GIKENCo., Ltd., Okayama, Japan) was accustomedto measure the manual muscle strength (MS)preoperatively and on PODs3, 7, 10, and 14 in a very manner the same as manual muscle testing. The ROM was measured using a goniometer. Preoperative MSand ROM were considered as $100 \%$, and the postoperative topreoperative MS ratio was calculated.

We further examined the femoral circumference at $10 \mathrm{~cm}$ above the superior margin of the patellae to evaluate soft tissue swelling. Preoperative femoral circumference was considered to be $100 \%$, and also the ratio of the postoperative to the preoperative circumference was calculated.

\section{Rescue dose}


All patients received oral celecoxib,200mg every $8 \mathrm{~h}$, for 14 days postoperatively. Intravenous or oral acetaminophen was provided if patients required additional analgesic drugs for postoperative pain. The number of rescue drugs administered for pain was recorded on PODs14.

\section{Radiographic measurements}

Preoperative and postoperative radiological assessments included anteroposterior views of the full-length lower extremities. We assessed the preoperative femorotibial angle (FTA) and postoperative implant coronal alignment, including the varus-valgus $(\alpha$ and $\beta$ ) angles of the femoral and tibial components $[15$, 16]. In this BCS TKA, the femoral component had a $3^{\circ}$ physiological line against the mechanical axis. Therefore, an a angle of $93^{\circ}$ was perpendicular to the mechanical axis.

\section{Statistical analysis}

A sample size calculation was performedusing the primary outcome measure of quadriceps muscle strength during the 14-day postoperative period [18], and28 patients in eacharm were required. All parameters are reported as mean \pm standard deviation. Differences between the groups were compared using Student's t-test or the Wilcoxon rank-sum test. These statistical analyses were performed using GraphPad Prism software (version 5.0; GraphPad Software, Inc., San Diego, CA, USA). Statistical significance was set at $\mathrm{P}<0.05$.

\section{Declarations}

\section{Author contributions}

$\mathrm{KM}$ and $\mathrm{HO}$ performed the operation. $\mathrm{KM}, \mathrm{HY}$, and $\mathrm{YN}$ collected the data. $\mathrm{KM}$, and $\mathrm{HO}$ analysed the data. $\mathrm{KM}$ wrote the paper. $\mathrm{KM}$, and $\mathrm{HA}$ prepared and confirmed the draft of manuscript.

Funding: None

Ethical approval: Our institute's ethics committee approved all of procedures performed in this study (approval number: 2019-238)

Informed consent: Informed consent was obtained from all participants.

\section{References}

1. Bourne RB, Chesworth BM, Davis AM, Mahomed NN, Charron KD. (2010) Patient satisfaction after total knee arthroplasty: who is satisfied and who is not? Clin OrthopRelat Res. 468(1):57-63.

2. Lotke PA, Ecker ML. (1977) Influence of positioning of prosthesis in total knee replacement. J Bone Joint Surg Am. 59:77-79.

3. Jeffery RS, Morris RW, Denham RA.(1991) Coronal alignment after total knee replacement. J Bone Joint Surg Br. 73:709-714. 
4. Lording T, Lustig S, Neyret P. (2017) Coronal alignment after total knee arthroplasty. EFORT Open Rev. 1:12-17.

5. Matsuda S, Kawahara S, Okazaki K, Tashiro Y, Iwamoto Y. (2013) Postoperative alignment and ROM affect patient satisfaction after TKA. Clin OrthopRelat Res 471:127-133.

6. Siebert W, Mai S, Kober R, Heeckt PF. (2002) Technique and first clinical results of robot-assisted total knee replacement. Knee. 9:173-180.

7. Jakopec M, Harris SJ, Rodriguez y, Baena F, Gomes P, Cobb J, Davies BL. (2001) The first clinical application of a 'hands-on' robotic knee surgery system. Comput Aided Surg. 6:329-339.

8. Banerjee S, Cherian JJ, Elmallah RK, Jauregui JJ, Pierce TP, Mont MA. (2015) Robotic-assisted knee arthroplasty. Expert Rev Med Devices. 12(6):727-35.

9. St Mart JP, Goh EL. (2021) The current state of robotics in total knee arthroplasty. EFORT Open Rev. 1;6(4):270-279.

10. Hampp EL, Chughtai M, Scholl LY, Sodhi N, Bhowmik-Stoker M, Jacofsky DJ, Mont MA. (2019) Robotic-Arm Assisted Total Knee Arthroplasty Demonstrated Greater Accuracy and Precision to Plan Compared with Manual Techniques. J Knee Surg. 32(3):239-250.

11. Bollars P, Boeckxstaens A, Mievis J, Kalaai S, Schotanus MGM, Janssen D. (2020) Preliminary experience with an image-free handheld robot for total knee arthroplasty: 77 cases compared with a matched control group. Eur J OrthopSurgTraumatol. 30(4):723-729.

12. Kayani B, Konan S, Tahmassebi J, Pietrzak JRT, Haddad FS. (2018) Robotic-arm assisted total knee arthroplasty is associated with improved early functional recovery and reduced time to hospital discharge compared with conventional jig-based total knee arthroplasty: a prospective cohort study. Bone Joint J. 100-B(7):930-937.

13. Marchand RC, Sodhi N, Khlopas A, Sultan AA, Harwin SF, Malkani AL, Mont MA. (2017) Patient satisfaction outcomes after robotic arm-assisted total knee arthroplasty: a short-term evaluation. J Knee Surg. 30:849-853.

14. Khlopas A, Sodhi N, Hozack WJ, Chen AF, Mahoney OM, Kinsey T, Orozco F, Mont MA. (2020) Patientreported functional and satisfaction outcomes after robotic-arm-assisted total knee arthroplasty: early results of a prospective multicenter investigation. J Knee Surg. 33:685-690.

15. Matsumoto K, Ogawa H, Fukuta M, Mori N, Akiyama H. (2018) Comparative Study for Alignment of Extramedullary Guides versus Portable, Accelerometer-Based Navigation in Total Knee Arthroplasty. J Knee Surg. 31(1):92-98.

16. Matsumoto K, Mori N, Ogawa H, Akiyama H.(2015) Accuracy of a novel extramedullary femoral alignment guide system in primary total knee arthroplasty. Arch Orthop Trauma Surg. 135(12):17438.

17. Eckhoff DG, Metzger RG, Vandewalle MV. (1995) Malrotation associated with implant alignment technique in total knee arthroplasty. Clin OrthopRelat Res (321):28-31

18. Matsumoto S, Matsumoto K, lida H. (2015) Transdermal fentanyl patch improves post-operative pain relief and promotes early functional recovery in patients undergoing primary total knee arthroplasty: 
a prospective, randomised, controlled trial. Arch Orthop Trauma Surg. 135(9):1291-7.

19. Paul HA, Bargar WL, Mittlestadt B, Musits B, Taylor RH, Kazanzides P, Zuhars J, Williamson B, Hanson W. (1992) Development of a surgical robot for cementless total hip arthroplasty. Clin OrthopRelat Res. 285, 57-66.

20. Park SE, Lee CT. (2007) Comparison of robotic-assisted and conventional manual implantation of a primary total knee arthroplasty. J Arthroplasty. 22:1054-1059.

21. Bhimani SJ, Bhimani R, Smith A, Eccles C, Smith L, Malkani A. (2020) Robotic-assisted total knee arthroplasty demonstrates decreased postoperative pain and opioid usage compared to conventional total knee arthroplasty. Bone Jt Open. 27;1(2):8-12.

22. Naziri Q, Cusson BC, Chaudhri M, Shah N V, Sastry A. (2019) Making the transition from traditional to robotic-arm assisted TKA: what to expect? A single-surgeon comparativeanalysis of the first-40 consecutive cases. J Orthop.16:364-368.

23. Jeon SW, Kim KI, Song SJ. (2019) Robot-assisted total knee arthroplasty does not improve long-term clinical and radiologic outcomes. J Arthroplasty. 34(08):1656-1661

24. Cho KJ, Seon JK, Jang WY, Park CG, Song EK. (2019) Robotic versus conventional primary total knee arthroplasty: clinical and radiological long-term results with a minimum follow-up of ten years. Int Orthop. 43(6):1345-1354.

25. Hampp EL, Sodhi N, Scholl L, Deren ME, Yenna Z, Westrich G, Mont MA. (2019) Less iatrogenic softtissue damage utilizing robotic-assisted total knee arthroplasty when compared with a manual approach: A blinded assessment. Bone Joint Res. 2;8(10):495-501.

26. Khlopas A, Chughtai M, Hampp EL, Scholl LY, Prieto M, Chang TC, Abbasi A, Bhowmik-Stoker M, Otto J, Jacofsky DJ, Mont MA. (2017) Robotic-Arm Assisted Total Knee Arthroplasty Demonstrated Soft Tissue Protection. SurgTechnol Int. 25;30:441-446.

27. Kayani B, Konan S, Huq SS, Tahmassebi J, Haddad FS. (2019) Robotic-arm assisted total knee arthroplasty has a learning curve of seven cases for integration into the surgical workflow but no learning curve effect for accuracy of implant positioning. Knee Surg Sports TraumatolArthrosc. 27(4):1132-1141.

28. Kaneko T, Kono N, Mochizuki Y, Hada M, Toyoda S, Musha Y. (2017) Bi-cruciate substituting total knee arthroplasty improved medio-lateral instability in mid-flexion range. J Orthop. 7;14(1):201-206.

\section{Tables}




\begin{tabular}{|c|c|c|c|}
\hline & Control group $(\mathrm{N}=54)$ & RA-TKA ${ }^{a}$ group $(N=56)$ & $P$ value \\
\hline Age & $73.4 \pm 7.8$ & $72.6 \pm 9.6$ & 0.9063 \\
\hline Sex (F:M) & $40: 15$ & $44: 14$ & \\
\hline Height (m) & $1.53 \pm 0.096$ & $1.52 \pm 0.096$ & 0.9364 \\
\hline Body weight $(\mathrm{kg})$ & $62.9 \pm 13.9$ & $60.7 \pm 9.6$ & 0.4496 \\
\hline $\mathrm{BMI}^{\mathrm{b}}$ & $26.8 \pm 4.4$ & $26.3 \pm 4.3$ & 0.4944 \\
\hline $\operatorname{FTA}^{\mathrm{C}}\left({ }^{\circ}\right)$ & $184.2 \pm 12.9$ & $185.0 \pm 8.9$ & 0.4922 \\
\hline
\end{tabular}

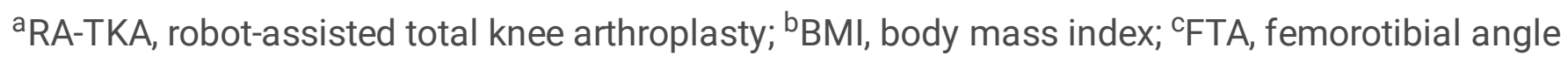

Table 2 Comparison of component alignment between the control and the RA-TKA group

\begin{tabular}{|lllc|}
\hline & $\begin{array}{l}\text { Control group } \\
(\mathbf{N}=54)\end{array}$ & $\begin{array}{l}\text { RA-TKA group } \\
(\mathbf{N}=56)\end{array}$ & $\begin{array}{l}\text { P } \\
\text { value }\end{array}$ \\
\hline $\begin{array}{l}\text { Coronal Alignment of Femoral Component }(\mathrm{a} \\
\text { angle) }\end{array}$ & $92.2^{\circ} \pm 2.1^{\circ}$ & $92.3^{\circ} \pm 1.1^{\circ}$ & 0.4232 \\
\hline $\begin{array}{l}\text { Coronal Alignment of Tibial Component }(\beta \\
\text { angle) }\end{array}$ & $89.6^{\circ} \pm 2.2^{\circ}$ & $89.6^{\circ} \pm 1.0^{\circ}$ & 0.4427 \\
\hline $\begin{array}{l}\text { Femoral Component Varus/Valgus: \% within } \\
2^{\circ}\end{array}$ & $92.6 \%$ & $92.9 \%$ & 0.9579 \\
\hline Tibial Component Varus/Valgus: \% within $2^{\circ}$ & $72.2^{\circ} \%$ & $96.4 \%$ & 0.0004 \\
\hline
\end{tabular}

aRA-TKA, robot-assisted total knee arthroplasty

\section{Figures}


a

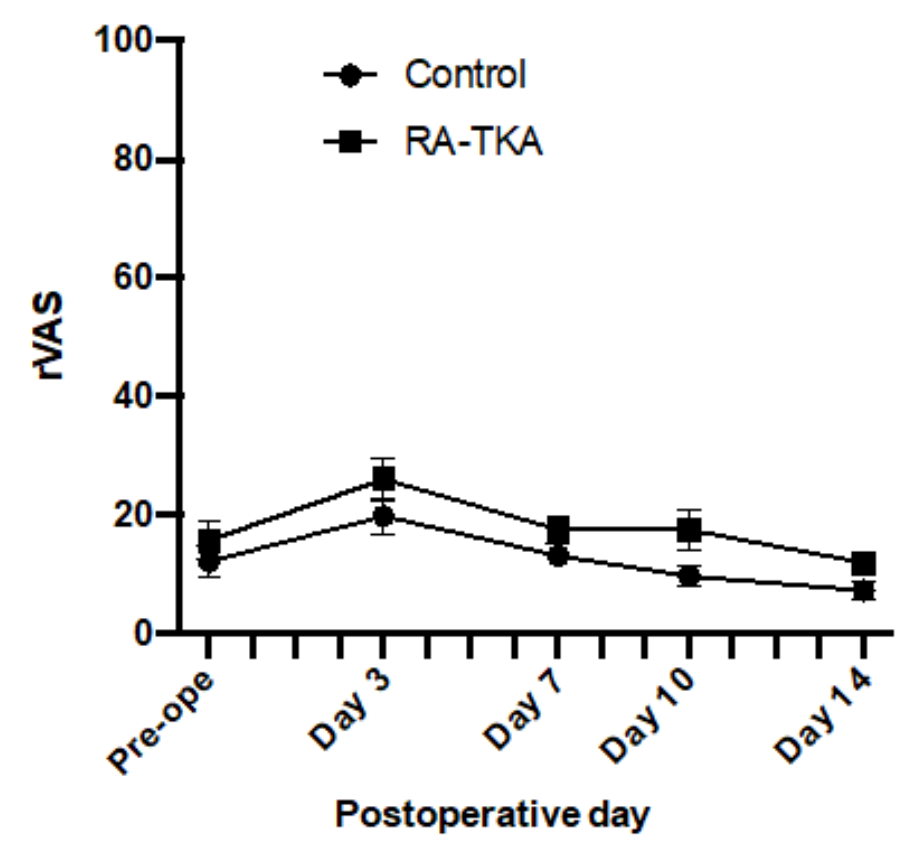

b

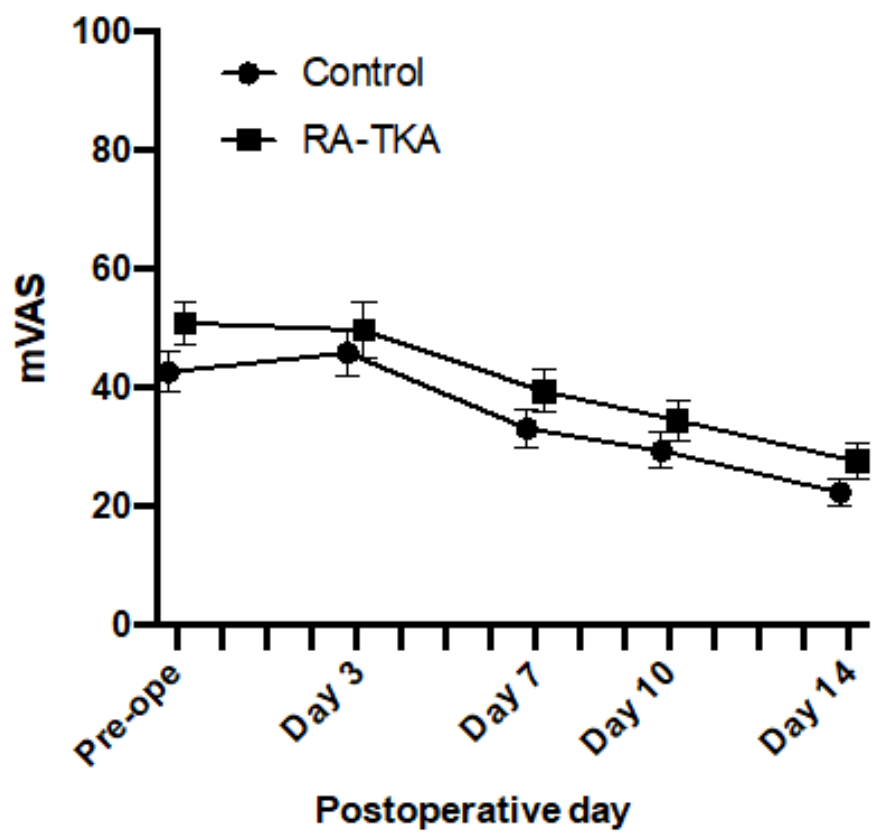

Figure 1

(a) Visual analogue scale at rest (rVAS). (b) Visual analogue scale during movement (mVAS). The rVAS and mVAS were comparable between the control and the robot-assisted total knee arthroplasty (RA-TKA) groups throughout the 14-day postoperative period

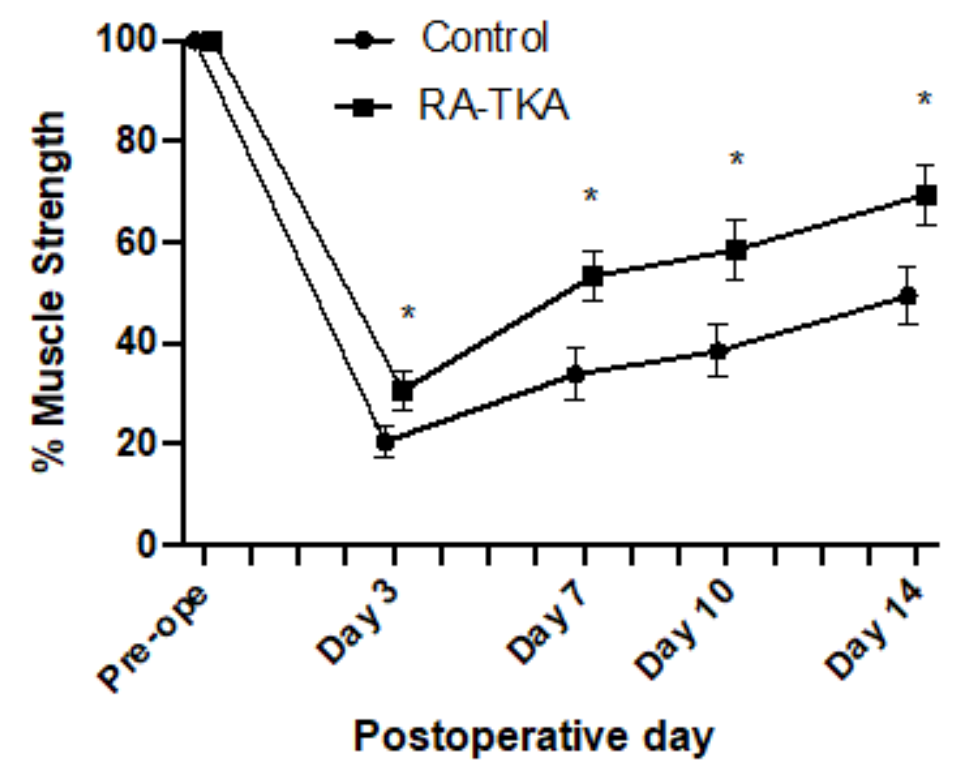

Figure 2 
Patients in the RA-TKA group showed significantly faster recovery of lower extremity muscle strength (\% muscle strength) than the control group on postoperative days (PODs) $3,7,10$, and 14

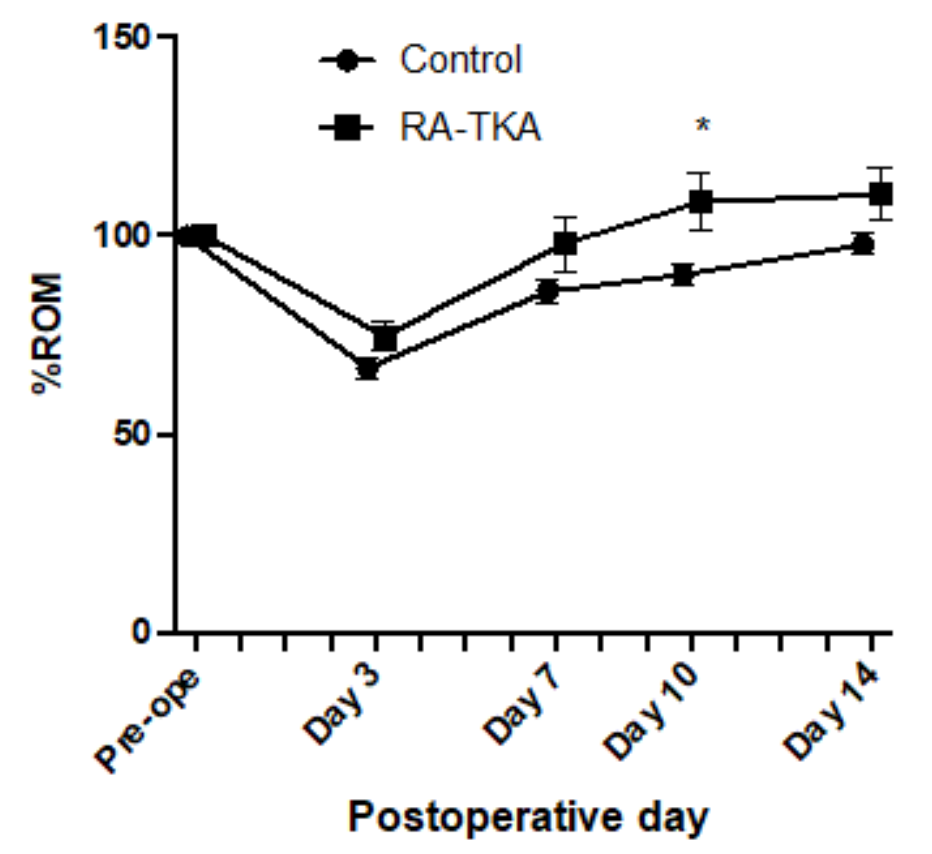

Figure 3

Patients in the RA-TKA group showed significantly better range of motion (ROM) recovery (\% ROM) than the control group, differing on POD 10

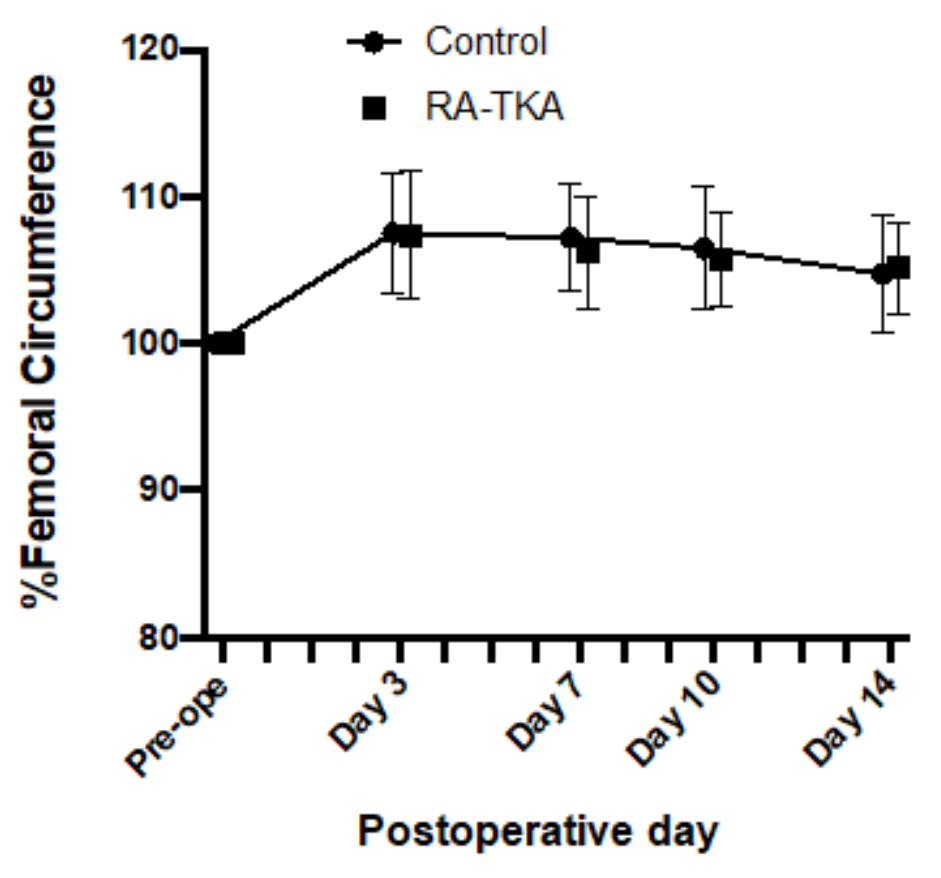

Figure 4

The femoral circumference (\%) was comparable in both groups through out the 14-day post operative period 
a

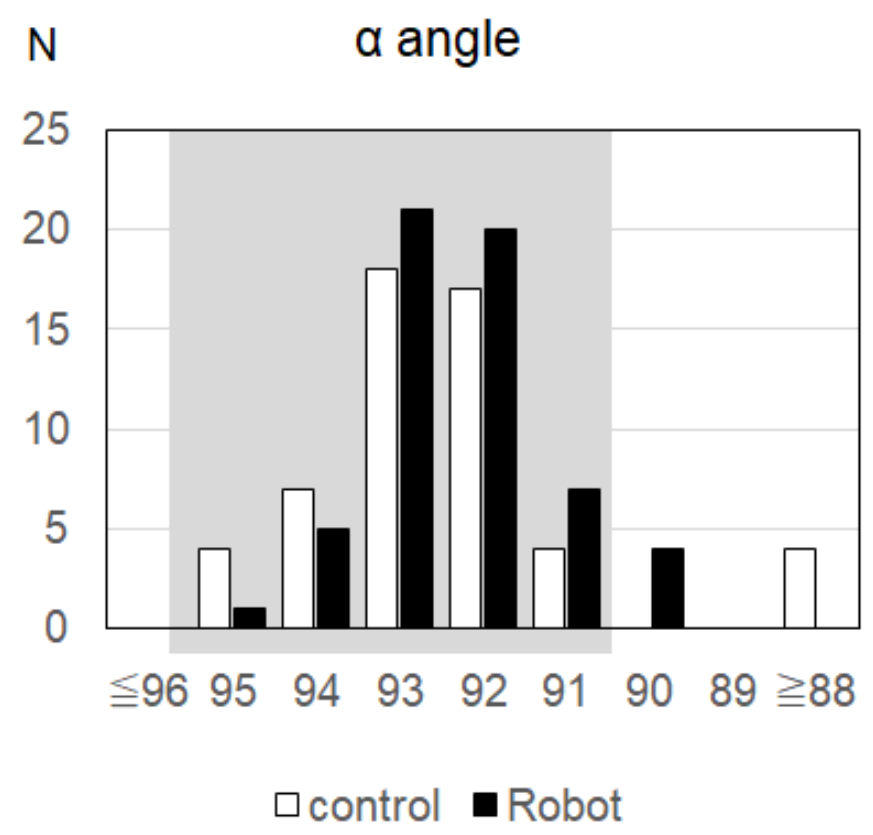

b

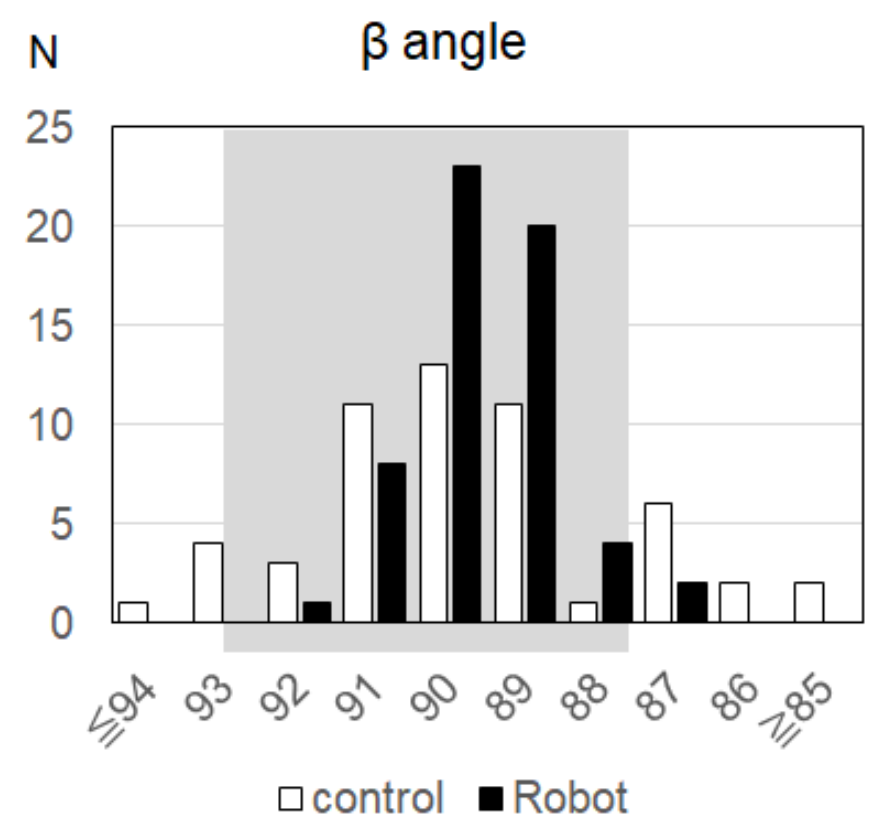

Figure 5

(a) Distribution of femoral a angles (coronal alignment) in the two groups. The grey area indicates less than $2^{\circ}$ of deviation from the mechanical axis in the coronal plane. (b) Distribution of tibial $\beta$ angles (coronal alignment) in the two groups. The grey area indicates less than $2^{\circ}$ of deviation from the mechanical axis in the coronal plane 